in serovar prevalence between men and women were detected. Finally, significant differences $(p=0.035)$ were detected when serovar distribution among patients with or without coinfection was studied: patients with an infection due to $\mathrm{D} / \mathrm{Da}$ had the highest coinfection rate $(75.0 \%)$, whereas coinfection rates among patients with serovars F, E, and G were $57.1 \%, 37.5 \%$, and $29.2 \%$, respectively see Abstract P3-S1.07 table 1.

Conclusions The present study contributed to increase the knowledge on serovar distribution of $C$ trachomatis in Italy.

\section{P3-S1.08 ARE THERE ACCEPTABLE ALTERNATIVES TO SYNDROMIC MANAGEMENT FOR THE DIAGNOSIS OF SEXUALLY TRANSMITTED INFECTIONS IN HIV POSITIVE KENYAN WOMEN?}

doi:10.1136/sextrans-2011-050108.408

${ }^{1} \mathrm{~V}$ Woo, ${ }^{2} \mathrm{C}$ R Cohen, ${ }^{3} \mathrm{E}$ A Bukusi, ${ }^{2} \mathrm{M}$ J Huchko. ${ }^{1}$ Stanford University, School of Medicine, Stanford, USA; ${ }^{2}$ University of California, San Francisco, USA; ${ }^{3}$ Kenya Medical Research Institute, Nairobi, Kenya

Background Control of sexually transmitting infections (STIs) is an important aspect of HIV prevention and care. Syndromic management for vaginal discharge, despite a recognised low sensitivity and specificity, is widely employed for STI management among HIV-1 infected women in resource-limited settings. In this pilot study, we evaluated the incremental increase in sensitivity and specificity of multiple, low-cost diagnostic strategies for non-ulcerative STIs diagnosed among HIV-1 infected women receiving care in Kenya. Methods This cross-sectional study was done among a cohort of HIV-1 infected women enrolled at Family AIDS and Care and Treatment Services (FACES) clinics in Kisumu, Kenya. During their routine clinic visit, participants reported any health complaint and later were asked about general vaginal symptoms (brief symptom ascertainment) and specific complaints of pruritis, odour and discharge (detailed symptom ascertainment). Clients were then examined for cervicitis and vaginal discharge, followed by specimen collection for STI testing. Results of Chlamydia trachomatis and Neisseria gonorrhoeae nucleic acid amplification test and Trichomonas vaginalis (TV) by wet mount was used as the gold standard for sensitivity and specificity calculations.

Results Of the 155 women who were screened between the ages of $23-53$ years, the prevalence of $N$ gonorrhoeae was $1.9 \%$ (3/155), TV was $6.4 \%(10 / 155)$ and no cases of $C$ trachomatis were detected. See Abstract P3-S1.08 table 1.

Conclusions Syndromic management had a very poor sensitivity for detecting STIs in HIV-1 infected women. The addition of specific questions about STI-related symptoms improved STI detection rates, while a speculum exam led to greater sensitivity and specificity. The feasibility and effectiveness of alternative approaches such

Abstract P3-S1.08 Table 1 Sensitivity and specificity of multiple diagnostic strategies for STI diagnosis among HIV-1 infected women receiving care at FACES

\begin{tabular}{|c|c|c|c|c|}
\hline $\begin{array}{l}\text { Evaluation } \\
\text { technique } N=155\end{array}$ & $\begin{array}{l}\text { Per cent } \\
\text { positive } \\
\text { results }\end{array}$ & $\begin{array}{l}\text { Sensitivity } \\
\text { for any STI* }\end{array}$ & $\begin{array}{l}\text { Specificity } \\
\text { for any STI* }\end{array}$ & $\begin{array}{l}\text { Positive } \\
\text { predictive } \\
\text { value }\end{array}$ \\
\hline $\begin{array}{l}\text { Self report (current } \\
\text { standard of care) }\end{array}$ & $3 \%(5)$ & $0(0)$ & $97 \%(137)$ & $0(0)$ \\
\hline $\begin{array}{l}\text { Intensified symptom } \\
\text { ascertainment, brief }\end{array}$ & $26 \%(40)$ & $23 \%(3)$ & $75 \%(107)$ & $8 \%$ \\
\hline $\begin{array}{l}\text { Intensified symptom } \\
\text { ascertainment, detailed }\end{array}$ & $26 \%(40)$ & $31 \%(4)$ & $75 \%(106)$ & $10 \%$ \\
\hline Gynaecologic exam & $9.0 \%(14)$ & $46 \%(6)$ & $94 \%(134)$ & $46 \%$ \\
\hline
\end{tabular}

*Includes $N$ gonorrhoeae, $C$ trachomatis and $T$ vaginalis. as routine use of speculum exams, and point-of-care testing for $T$ vaginalis should be explored to improve the management of STIs among HIV-1 infected women in similar low-resource settings.

\section{P3-S1.09 VALIDATION OF COBAS ${ }^{\circledR} 4800$ HPV AND CT/NG TEST IN CLINICAL SAMPLES}

doi:10.1136/sextrans-2011-050108.409

${ }^{1} \mathrm{M}$ Basaras, ${ }^{1} \mathrm{E}$ Arrese, ${ }^{2} \mathrm{D}$ Andia, ${ }^{2} \mathrm{~S}$ Hernaez, ${ }^{2} \mathrm{~V}$ Esteban, ${ }^{3} \mathrm{M}$ Camara, ${ }^{4} \mathrm{R}$ Cisterna ${ }^{1}$ Universidad del País Vasco, Leioa-Bizkaia, Spain; ${ }^{2}$ Hospital de Basurto, Spain; ${ }^{3}$ Cantro de ETS Bombero, Etxaniz, Spain; ${ }^{4}$ Hospital de Basurto, Universidad del País Vasco, Spain

Background Roche cobas ${ }^{\circledR} 4800$ system performs sample preparation, real-time PCR amplification and detection using an internal control in a single tube. The cobas ${ }^{\circledR} 4800$ human papillomavirus (HPV) test is a multiplex assay that can detect HPV 16, HPV 18 and 12 other high-risk (12-HR) carcinogenic HPV genotypes. We compared this HPV test with the Linear Array (LA) HPV genotyping assay (Roche Molecular System). Therefore, this system can simultaneously detect Chlamydia trachomatis (CT) and Neisseria gonorrhoeae (NG) in urine and swab specimens, and it was evaluated and compared with routine techniques in our clinical laboratory.

Methods 462 clinical samples collected in PresevCyt ${ }^{\circledR}$ liquid media were used for the HPV test study and compared using cobas ${ }^{\circledR} 4800$ HPV test and LA-HPV. There were also included 688 clinical samples for CT and NG testing (206 urine samples only and 241 urine and swab specimens respectively) and compared with a real-time PCR assay for CT and bacteria culture for NG.

Results For the HPV analysis, 439 out of 462 samples examined (95\%) showed positive (160 cases) and negative (302 cases) concordat results; the remaining $3(0.65 \%)$ were invalid by the cobas ${ }^{\circledR} 4800$ system. The positive samples were distributed: 26 samples of HPV 16, 4 of HPV 18, 110 of 12-HR and 20 samples with mixed infection (15 were 12-HR + HPV 16, four 12-HR+HPV 18 and one HPV 16+18). For CT/NG total analysis, there were only three invalid samples $(0.43 \%)$ and only $4(0.58 \%)$ discordant results. For the 206 urine samples, there were 19 CT positive, eight NG positive and two mixed CT and NG infections. These positive samples were from male with Chlamydia contact, urethritis and persons who was to STI control. For urine and swab specimens (241 of each), there were a total correlation between both types of samples. In total, there were $5 \%$ of positive samples corresponding to 13 CT positive, two NG positive and four mixed CT and NG infections. These positive samples presented clinical manifestations as urethitis or were women to get in touch with Chlamydia infected person.

Conclusions the cobas ${ }^{\circledR} 4800$ system is an easy system for cervical HPV screening and to detect simultaneously CT and NG in a single tube. These test and our lab techniques correlated well in this analysis. Moreover, in the case of CT/NG test the correlation between urine and swab specimens was total, therefore to use urine as clinical sample to detect these two bacteria could be easier than to use swab specimens.

\section{P3-S1.10 USING ELECTRONIC MEDICAL RECORD DATA TO GUIDE EXPEDITED PARTNER THERAPY IMPLEMENTATION IN AN URBAN STD CLINIC SYSTEM, 2009}

doi:10.1136/sextrans-2011-050108.410

${ }^{1} \mathrm{~T}$ Mulder, ${ }^{2} \mathrm{~K}$ Johnson, ${ }^{2} \mathrm{~A}$ Lifflander, ${ }^{2} \mathrm{~J}$ Schillinger, ${ }^{2} \mathrm{M}$ Rogers, ${ }^{2} \mathrm{~S}$ Blank. ${ }^{1}$ US Centers for Disease Control and Prevention, Atlanta, USA; ${ }^{2}$ NYC Department of Health, US Centers for Disease Control and Prevention, New York, USA

Background Expedited partner therapy (EPT) is the practice of providing treatment without a clinical assessment to sex partners of 